\begin{tabular}{|c|c|c|}
\hline & Int.J.Curr.Microbiol.App.Sci (2021) 10(10): 176-184 & \\
\hline & $\begin{array}{l}\text { International Journal of Current Microbiology and Applied Sciences } \\
\text { ISSN: 2319-7706 Volume } 10 \text { Number } \mathbf{1 0}(\mathbf{2 0 2 1 )} \\
\text { Journal homepage: http://www.ijcmas.com }\end{array}$ & 30 \\
\hline $\begin{array}{l}\text { EXCELLENT } \\
\text { PUBLISHERS }\end{array}$ & & \\
\hline
\end{tabular}

\title{
Coproduction of Extended Spectrum, AmpC and Metallo $\beta$ - Lactamases in Pseudomonas aeruginosa Isolates from a Super Speciality Center
}

\author{
Ashna Bhasin*, Poonam Loomba, Abha Sharma, Bibhabati Mishra and Ashish Bajaj \\ Department of Microbiology, Govind Ballabh Pant Institute of Postgraduate Medical \\ Education and Research, Delhi, India \\ *Corresponding author
}

\section{A B S T R A C T}

\begin{tabular}{|l|}
\hline K e y w o r d s \\
Antibiotics, \\
Beta-Lactamase, \\
Community \\
acquired infections, \\
ESBLs \\
\hline Article Info \\
\hline $\begin{array}{l}\text { Accepted: } \\
\text { 10 September } 2021 \\
\text { Available Online: } \\
\text { 10 October } 2021\end{array}$ \\
\hline
\end{tabular}

\section{Keywords}

Antibiotics,

Beta-Lactamase,

Community

acquired infections, ESBLs

Pseudomonas aeruginosa ( $P$. aeruginosa) is one of the leading causes of hospital as well as community acquired infections. They're strenuous to treat as most of isolates exhibit various degrees of beta- lactamase mediated resistance to majority of the betalactam antibiotics. Single isolate can express multiple $\beta$ - lactamase enzymes, further limiting the treatment options. Therefore, this study was outlined to research the coexistence of various beta-lactamase enzymes in clinical isolates of $P$. aeruginosa. The aim of the study was to detect the co-prevalence of Extended Spectrum Beta lactmases (ESBL), AmpC and Metallo $\beta$-Lactamases (MBL) in Pseudomonas aeruginosa isolates from a superspeciality center. Fifty clinical isolates of $P$. aeruginosa were tested for the presence of AmpC beta-lactamase, extended spectrum beta- lactamase (ESBL) and metallo beta-lactamase (MBL) enzyme. Discernment of AmpC beta-lactamase was performed by disk antagonism while ESBL detection was done by the combined disk diffusion method as per Clinical and Laboratory Standards Institute (CLSI) guidelines and MBL were detected by the Imipenem EDTA disk potentiation test. Eleven of 50 (22\%) isolates were confirmed to be positive for AmpC and Extended spectrum beta lactamases. Co-production of AmpC along side ESBL and MBL was reported in $12 \%$ isolates. The study shows the high prevalence of multidrug resistant $P$. aeruginosa producing beta-lactamase enzymes of diverse mechanisms. Consequently, formulation of a correct antibiotic policy and taking measures to restrict the indiscriminative use of cephalosporins and carbapenems should be taken to mitigate the emergence of this multiple beta-lactamase producing

\section{Introduction}

The rise in antibiotic resistance among gramnegative bacteria may be a classic example of how a bacteria can procure, maintain, and express new genetic information which will confer resistance to at least one or several other antibiotics. A consensus appears to prevail that quinolones and broad-spectrum $\beta$ lactam resistance are increasing in members of the Enterobacteriaceae which is the reason why treatment regimes for the eradication of 
Pseudomonas aeruginosa ( $P$. aeruginosa) infections are getting increasingly limited (Balan, et al., 2013). The advent of carbapenems within the 1980s heralded a replacement treatment option for serious bacterial infections.

But now Carbapenem resistance is observed in Enterobacteriaceae and is becoming common also in $P$. aeruginosa. $P$. aeruginosa is one of the main etiological agents of nosocomial infection. They're extremely difficult to treat as most of them exhibit varying degrees of innate resistance. Acquired resistance is mediated by the assembly of chromosomal and plasmid-mediated AmpC beta-lactamases, extended-spectrum beta-lactamases (ESBLs), and Metallo-beta-lactamases (MBLs).

AmpC beta-lactamases play a crucial role in the resistance of Gram-negative bacilli. AmpC enzymes are grouped into Ambler Class C classification (Balan K et al., 2013). These enzymes confer a high level of resistance to several beta-lactam antibiotics including the third-generation cephalosporins and cephamycins. With the gradual increase in the occurrence of those multiple beta-lactamase enzymes, early detection is crucial for the initiation of proper antibiotic therapy and infection control policy.

The aim of this study to detect the coprevalence of ESBL, AmpC, and Metallo $\beta$ Lactamases in Pseudomonas aeruginosa isolates from a super-specialty center

\section{Materials and Methods}

The study was conducted at the Department of Microbiology of a teaching Superspeciality hospital.

\section{Bacterial isolates and characterization}

A total of fifty random sequenced isolates of
$P$. aeruginosa were isolated from varied clinical samples received for routine laboratory investigations from patients getting to the hospital from December 2018 to June 2019. These isolates were identified as $P$. aeruginosa by standard conventional methods.

\section{Antimicrobial susceptibility testing}

The antimicrobial susceptibility of those isolates was performed on Mueller-Hinton agar (MHA) using commercial antibiotic discs (Hi-Media Laboratories Ltd, Mumbai) by the standard quality Kirby-Bauer disc diffusion technique and interpreted as per CLSI recommendation.[2] The antibiotics used were gentamicin $(10 \mu \mathrm{g}), \quad$ amikacin $(30 \mu \mathrm{g})$, tobramycin $(10 \mu \mathrm{g})$, ciprofloxacin $(5 \mu \mathrm{g})$, ceftazidime $(30 \mu \mathrm{g})$, levofloxacin $(5 \mu \mathrm{g})$, piperacillin/tazobactam $\quad(100 / 10 \mu \mathrm{g})$, meropenem $(10 \mu \mathrm{g})$, imipenem $(10 \mu \mathrm{g})$, colistin $(10 \mu \mathrm{g})$, cefepime $(30 \mu \mathrm{g})$, netilimicin $(30 \mu \mathrm{g})$, ticarcillin clavulanic acid $(75 / 10 \mu \mathrm{g}) . \quad P$. aeruginosa ATCC 27853 was used as control.

\section{ESBL detection}

Isolate that showed immune to a minimum of one among the third generation cephalosporins were tested for ESBL production by phenotypic confirmation test suggested by CLSI.

\section{CLSI phenotypic confirmation test}

A McFarland 0.5 standard suspension of the $P$. aeruginosa isolate was inoculated onto the MHA plate. Ceftazidime $(30 \mu \mathrm{g})$ and ceftazidime/clavulanic acid $(30 / 10 \mu \mathrm{g})$, discs were placed on inoculated MHA plate at a distance of $30 \mathrm{~mm}$ aside from center to center. The culture was incubated at $37^{\circ} \mathrm{C}$ overnight. The observation of a $\geq 5 \mathrm{~mm}$ increase in zone diameter of ceftazidime/clavulanic acid disc than ceftazidime disc alone was considered ESBL producer (CLSI 2018). 


\section{AmpC $\beta$-lactamase detection}

Cefoxitin-resistant isolates were screened for AmpC $\beta$-lactamase production.

\section{Inducible AmpC detection}

\section{Disc antagonism test}

A McFarland 0.5 standard suspension of the isolate was inoculated onto the MHA plate. Cefotaxime $(30 \mu \mathrm{g})$ and cefoxitin $(30 \mu \mathrm{g})$ discs were placed $20 \mathrm{~mm}$ aside from center to center. The culture was incubated at $37^{\circ} \mathrm{C}$ nightlong. Isolates showing blunting of the cefotaxime zone of inhibition adjacent to the cefoxitin disc indicate inducible AmpC production (Padiyath, et al., 2013).

\section{MBL detection}

\section{Screening for MBLs}

Isolates immune to meropenem or imipenem were screened for MBL production. All audition positive isolates were subjected to corroboratory check (Padiyath, et al., 2013).

\section{Disc potentiation test}

The standard suspension of the check isolate was inoculated onto the MHA plate. A $0.5 \mathrm{M}$ resolution of EDTA was prepared by dissolving $186.1 \mathrm{gm}$ of disodium EDTA $2 \mathrm{H}_{2} \mathrm{O}$ in $1000 \mathrm{~mL}$ of distilled water and $\mathrm{pH}$ was adjusted to eight by victimization $\mathrm{NaOH}$. The mixture was sterilized by autoclaving. Two $10 \mu \mathrm{g}$ imipenem disc was placed $20 \mathrm{~mm}$ aside from center to center onto the MHA plate. To at least one disc of imipenem, $5 \mu 1$ of $0.5 \mathrm{M}$ EDTA was added. The zone of inhibition of imipenem and imipenem/EDTA discs were compared once 16-18 hours of incubation at $35^{\circ} \mathrm{C}$. A rise within the zone of inhibition by a minimum of $7 \mathrm{~mm}$ with imipenem/EDTA disc than imipenem alone was thought about as
MBL positive (Padiyath, et al., 2013).

\section{Results and Discussion}

\section{Bacterial Isolates}

A total of 50 non-repetitive and non redundant clinical isolates of $P$. aeruginosa (bile 20, mucous trap 13, pus 7 , blood 6 , tissue 2 , cerebrospinal fluid 2, and bronchoalveolar lavage 1) from the inpatient department (IPD) and the outpatient department (OPD) were included in this study. Out of which, 29 (58\%) strains were isolated from male patients and $21(42 \%)$ from female patients. The majority of the isolates $(96 \%)$ were from the inpatient department (IPD), while $4 \%$ of isolates were from the outpatient department (OPD) of the hospital. Thirty-three (66\%) isolates were from patients admitted to intensive care units.

Associated risk factors for the development of $P$. aeruginosa were studied in all the patients. Eighteen (36\%) patients were found to be immunocompromised or having comorbid conditions like diabetes mellitus, hypertension, tuberculosis, etc. Apart from this, the patients were also studied for other most common risk factors commonly seen in $P$. aeruginosa shown in table 1.Except for 6 patients, all other patients were already on multiple antibiotics.

\section{Antibacterial Susceptibility Profile}

Among tested antibiotics, Seventy-six percent of isolates were resistant to fluoroquinolone (ciprofloxacin and levofloxacin). The beta lactams were found to be less effective in $P$. aeruginosa with $70 \%$ resistance mediated towards piperacillin and tazobactam, whereas more than half of the isolates were resistant to other beta-lactam antibiotics. Aminoglycosides (gentamicin, netilmicin, and tobramycin) also showed a resistance rate up to $51.4 \%$, whereas amikacin showed a higher 
resistance rate of $58 \%$. Colistin was found to be the best drug for the treatment of infections caused by $P$. aeruginosa, as its resistance was not seen in this study (Figure 1).

Coproduction of ESBL/AmpC/MBL was presented in (Table 3). Eleven isolates (22\%) were positive for both ESBL and AmpC while six isolates (12\%) were positive for AmpC and MBL. Coproduction of ESBL and MBL was detected in $9(18 \%)$ of the isolates. All three enzyme production were seen in $6(12 \%)$ isolates.

Many patients infected with $P$. aeruginosa having coproduction of various $\beta$ - lactamases developed serious complications. Six patients (12\%) developed bloodstream infections, five patients (10\%) developed pneumonia/VAP and urinary Tract infection (UTI) each and one developed (2\%)surgical site infection. However, ten patients $(20 \%)$ eventually succumbed to this infection.

Pseudomonas aeruginosa is the commonest pathogen causing nosocomial infection mostly in immunocompromised patients. These infections include bacteremia, endocarditis, meningitis, otitis, osteomyelitis, burn, wound infection, etc. It's mostly present in the hospital environment (sinks, drains, respirators, humidifiers, and disinfectant solutions, etc.). Most of the hospital isolates are multi-drug resistant organisms (MDROs). Carbapenems are the drug of choice for MDR strains of $P$. aeruginosa. Nowadays, Carbapenem resistance has been observed in $P$. aeruginosa putting more pressure on reserved class drugs like Colistin for the treatment of such infections.

In the present study, we took 50 non repeated isolates of $P$. aeruginosa. Maximum number of $P$. aeruginosa was isolated from bile samples $(40 \%)$ followed by mucous $\operatorname{trap}(26 \%), \quad \operatorname{pus}(14 \%), \quad \operatorname{blood}(12 \%)$,
Tissue(4\%), cerebrospinal fluid (2\%) and bronchoalveolar lavage (2\%). However, Chander A et al., Senthamarai S et al., and Padiyath $\mathrm{S}$ et al., reported $P$. aeruginosa mostly in wound infections followed by respiratory tract infections in their studies. The probable reason for this might be that our place of study may be a super-specialty center and here general samples are less common. $P$. aeruginosa was more commonly isolated from male patients $(58 \%, 29 / 50)$ than female patients $(42 \%, 21 / 50)$ within the present study. Similar observations were made by Pal et al., Senthamarai S et al., \& Ranjan S et al., Adults starting from age bracket 55-65 years were most ordinarily affected age bracket which is analogous to other studies conducted in our institute (Ranjan et al., 2014) (Chander et al., 2013) (Senthamarai et al.,2014).

The majority of the patients were from the inpatient departments (96\%) while outpatients constituted only $4 \%$ of the cases. Out of the inpatients, $66 \%$ of patients belonged to medical care units. The majority of the patients belonged to gastroenterology and gastro surgery departments. Thirty-six percent of patients were immunocompromised or were having other comorbid conditions like DM, hypertension, tuberculosis, etc. The patients were also studied for other commonest risk factors related to $P$. aeruginosa, presence of an indwelling catheter $(40 \%)$ and prolonged ICU stay / bed ridden patients (46\%) constituted the main risk factor. The majority of the patients $(88 \%)$ were on multiple antibiotics.

Colistin was found to be the most beneficial drug for the treatment of infections caused by $P$. aeruginosa, as its resistance wasn't seen during this study. Similar findings were seen by Padiyath et al., and Choudhary V et al., (Padiyath, et al., 2013) (Choudhary, et al., 2018). Varaiya et al., depicted very low $(57.5 \%)$ colistin susceptibility. This difference 
might be thanks to the study environment under which the study was performed. Colistin remains the mainstay of treatment for multidrug-resistant $P$. aeruginosa (Kumar, et al., 2018). Imipenem, Meropenem which were initially very active against $P$. aeruginosa, showed marked resistance $(74 \%)$, results of which are in concordance with previous other studies (Kumar, et al., 2018). High resistance to Ceftazidime $(72 \%)$ was observed in our study, but similarly, $70 \%$ and $78 \%$ strains immune to Ceftazidime are reported by Behare et al., and Kumar et al., (Behra, et al., 2008) (Kumar, et al., 2014). Among tested antibiotics, seventy-six percent isolates were immune to fluoroquinolone (ciprofloxacin and levofloxacin). The beta-lactams were found to possess limited efficacy in $P$. aeruginosa with $70 \%$ resistance mediated towards piperacillin and tazobactam, whereas quite half the isolates were immune to other beta-lactam antibiotics. Aminoglycosides (gentamicin, netilmicin, and tobramycin) also showed a resistance rate up to $51.4 \%$, whereas amikacin showed a higher resistance rate of $58 \%$. Similar finding were seen in a study done by Gupta R et al., (Gupta, et al., 2016). Resistance to ceftazidime is especially mediated by the assembly of $\beta$-lactamases like extended-spectrum $\beta$ - lactamase, Metallo- $\beta$ lactamase and infrequently AmpC $\beta$ lactamases. The high resistance to cephalosporins exhibited by $P$. aeruginosa during this study might be thanks to the selective pressure of cephalosporin usage (Padiyath, et al., 2013).

ESBLs are Class A $\beta$ - lactamases, which hydrolyze all cephalosporins, penicillins, and monobactams but not cephamycins or carbapenems. They're inhibited in vitro by clavulanate. ESBL production was seen in $42 \%$ isolates. Variable incidence of ESBL production has been seen in our country starting from as low as 13 you must as high as $80 \%$. A Lower prevalence of $20-25 \%$ ESBL producing isolates have been reported from different parts of India like Nagpur (Tankhiwale, et al., 2016) and Lucknow (Shaikh, et al., 2015) and an even higher rate of $59.45 \%$ has been reported from Uttar Pradesh (Haider, et al., 2014) and $57.75 \%$ from Bangalore (Easwaran, et al., 2016). The high frequency of ESBLs in $P$. aeruginosa is thanks to the horizontal spread of genes. This varied ESBL production might be thanks to the nonjudicial use of antibiotics or thanks to environmental influence.

AmpC $\beta$ lactamases are cephalosporinases that aren't inhibited by clavulanic acid. Within the current study, the AmpC production was seen in $16 \%$ isolates. Compared to those findings other studies have reported a better prevalence of AmpC producers while some have reported lower prevalence. Studies from Pudduchery, Gujarat, and Himachal Pradesh reported a prevalence of 20-35\%, whereas Upadhyay S et al., reported $59.40 \%$ and Gupta, et al., reported $51.4 \%$ AmpC producing $P$. aeruginosa. This wide selection of prevalence of the AmpC producers in various studies might be thanks to the differences within the geographical distribution, which can have cause variations within the prevalence of the $\beta$ - lactamases present within the different organisms (Choudhary, et al.,2018).

The only $\beta$ - lactams active against co- AmpC and ESBL producers are carbapenems. Lately, carbapenem resistance is increasing in $P$. aeruginosa. This has been attributed to decreased outer membrane permeability which is thanks to the loss of Opr D porin, upregulation of active efflux pump system, alteration of penicillin-binding protein, or by the production of carbapenemases (Padiyath, et al., 2013). 
Table.1 Risk factors associated

\begin{tabular}{|c|c|}
\hline Chronic obstructive pulmonary disease & $\mathbf{1}$ \\
\hline \multicolumn{1}{|c|}{ Burn } & 0 \\
\hline \multicolumn{1}{|c|}{ Cystic fibrosis } & 0 \\
\hline Length of hospital stay(ICU/ bedridden) & 23 \\
\hline \multicolumn{1}{|c|}{ Mechanical ventilation } & 16 \\
\hline $\begin{array}{l}\text { Molignant disease } \\
\text { Age }>\text { 86 yrs } \\
\text { Antibiotic treatment for previous } 3 \\
\text { months }\end{array}$ & 3 \\
\hline Indwelling devices & 2 \\
\hline Physical disabilities & 7 \\
\hline
\end{tabular}

Table.2 Proportion of ESBL, AmpC and MBL production in P.aeruginosa isolates.

\begin{tabular}{|c|c|c|}
\hline Enzyme & $\begin{array}{c}\text { Number of Isolates of } \\
\text { Pseudomonas aeruginosa } \\
\text { showing the presence of } \\
\text { enzyme (N=50) }\end{array}$ & $\begin{array}{c}\text { Number of Isolates of } \\
\text { Pseudomonas aeruginosa } \\
\text { showing the presence of } \\
\text { enzyme(N=50) }\end{array}$ \\
\hline AmpC & 8 & $16 \%$ \\
\hline ESBL & 21 & $42 \%$ \\
\hline MBL & 21 & $42 \%$ \\
\hline
\end{tabular}

Table.3 Coproduction of various $\beta$ - lactamases

\begin{tabular}{|c|c|c|}
\hline Enzyme & $\begin{array}{c}\text { Number of Isolates of } \\
\text { Pseudomonas aeruginosa } \\
\text { showing the presence of } \\
\text { enzyme (N=50) }\end{array}$ & $\begin{array}{c}\text { Number of Isolates of } \\
\text { Pseudomonas aeruginosa } \\
\text { showing the presence of } \\
\text { enzyme (N=50) }\end{array}$ \\
\hline AmpC+ ESBL & 11 & $22 \%$ \\
\hline AmpC+MBL & 6 & $12 \%$ \\
\hline ESBL+MBL & 9 & $18 \%$ \\
\hline AmpC +ESBL+MBL & 6 & $12 \%$ \\
\hline $\begin{array}{c}\text { No resistance/other } \\
\text { resistance }\end{array}$ & 18 & $36 \%$ \\
\hline
\end{tabular}


Fig.1 Antibiotic susceptibility pattern of Pseudomonas aeruginosa

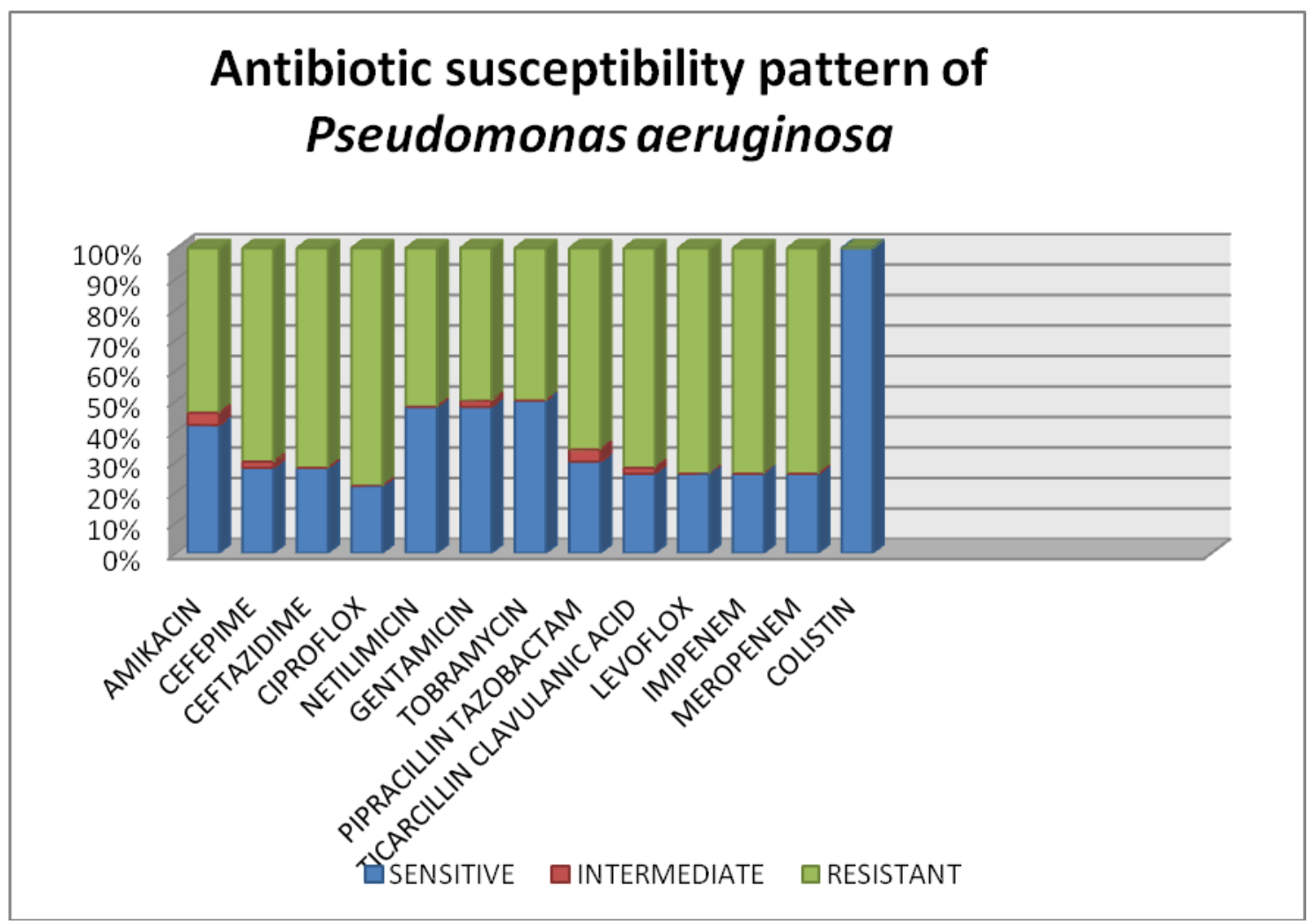

Metallo $\beta$ - lactamases are carbapenemases, which require zinc divalent cation, as a cofactor for enzyme activity. They're ready to hydrolyze all $\beta$ - lactams except monobactam and are universally inhibited by ethylenediamine tetra ethanoic acid (EDTA) also as other chelating divalent cations. The incidence of MBL producers within the present study was $42 \%$ which correlated well with other studies as $33.3 \%, 41 \%$ respectively (Anuradha et al., 2014) (Chate et al., 2014). Although, few studies reported a quite high incidence of MBL producing $P$. aeruginosa strains as $61.5 \%$ and $69.5 \%$ (Behra B et al., 2008) (Sharma et al., 2010)

Coproduction of ESBL/AmpC/MBL was also observed in our study. Eleven isolates (22\%) were positive for both ESBL and AmpC while six isolates (12\%) were positive for AmpC and MBL. Coproduction of ESBL and MBL was detected in $9(18 \%)$ of the isolates. All the three enzymes were seen in $6(12 \%)$ isolates while 36\% showed the presence of no resistance. Studies from various parts of India have reported prevalence rates of MBL and ESBL/AmpC co-existence between 4-27\% (4\% by Padiyath et al., $7.77 \%$ by Minhas, et al., $10.12 \%$ by Tankhiwale et al., $18.8 \%$ by Rawat et al., $27.72 \%$ by Upadhyay et al.,) (Choudhary et al., 2018).

Many patients affected by $P$. aeruginosa having coproduction of varied $\beta$ - lactamases developed serious complications. Six patients (12\%) developed blood-stream infection, five patients $(10 \%)$ developed pneumonia/VAP and tract infection (UTI) each and one developed (2\%) surgical site infection. However, ten patients (20\%) eventually succumbed to the present infection. Multiple $\beta$-lactamase producing $P$. aeruginosa may cause major therapeutic failure and pose a big clinical challenge if they continue to be 
undetected. Since these organisms might carry other drug-resistant genes, the sole available treatment option remains is that the administration of colistin which is potentially toxic. The potential limitation of this study is that molecular analysis and characterization of ESBL, AmpC, and MBL types weren't administered. Though Polymerase Chain Reaction (PCR) is taken into account as the gold standard for detection of newer $\beta$ lactamases however it's very costly and requires expertise and is beyond the scope of routine Clinical Microbiology Laboratories in India. Considering the existence of numerous sorts of newer $\beta$-lactamases, the most disadvantage of PCR is that it requires tailormade DNA primers, cannot differentiate between variants, and should not detect new variants. Similarly, E tests for ESBL, Amp C, and MBL are often used but they're also very costly and can't be used routinely. Hence, detection of resistance using simple disc methods described within the present study should be encouraged.

In the present study deduce that Pseudomonas aeruginosa being a stubborn multidrugresistant pathogen leaves colistin as the last resort for treatment of life-threatening infections in the hospital.

Increasing occurrence of multiple $\beta$ lactamases in clinical isolates of $P$. aeruginosa could lead to therapeutic failure. In routine circumstances, routine culture, and susceptibility tests should be performed to detect the emergence of resistance to Pseudomonas aeruginosa as early as possible. Attention by the hospital infection control team is essential to implement stringent preventive measures to contain the spread of the infection and promote the judicious use of antimicrobial agents.

Antibiotic resistance is increasing at an alarming rate, leading to increased morbidity, mortality, and treatment costs. A key factor in the development of antibiotic resistance is the inappropriate use of antibiotics. The medical fraternity needs to understand that antibiotics constitute a precious and finite resource. Until and unless responsive efforts are made to contain the peril of drug resistance, multi-drug resistant organisms, uncurable by every known antibiotic, may emerge victorious, reversing the medical progress made by mankind and throwing us back to the preantibiotic era.

Hence, early detection of $\beta$-lactamase production can benefit the implementation of proper antibiotic therapy and infection control policies.

\section{References}

Anuradha B, Afreen U, Praveena M. Evaluation of antimicrobial susceptibility pattern of Pseudomonas aeruginosa with special reference to MBL production in a tertiary care hospital. Glob J Med Res. 2014;14(7):23-8.

Balan K, Ali A. Comparison of different phenotypic methods for AmpC detection from rural hospital. Int J Cur Tr Res 2013;2: 105-7.

Behra B, Mathur P, Das A et al., An evaluation of four different phenotypic techniques for detection of metallo beta lactamases producing Pseudomonas aeruginosa. Indian J Med Microbiol. 2008; 26 (3): 233-7.

Chander A, Raza M S. Antimicrobial susceptibility patterns of $P$. aeruginosa clinical isolates at a tertiary care hospital in Kathmandu, Nepal. Asian J Pharm 25. Clinical Res 2013; 6:235-8.

Chate S, Watve S, Dardi C et al., Antibiotic resistance pattern of Pseudomonas aeruginosa with special reference to Imipenem and metallo beta lactamase production. Indian J Basic Appl Med Res. 2014; 4 (1): 117-122.

Choudhary Vinita., Pal Nita and Hooja Saroj. Phenotypic detection of ESBL, AmpC and MBL $\beta$-lactamases among clinical isolates 
of Pseudomonas aeruginosa in a tertiary care hospital of north India. Int $\mathbf{J}$ Community Med Public Health 2018; 4:3902-3906.

Clinical and Laboratory Standards Institute (CLSI), Performance Standards for Antimicrobial Susceptibility Testing; Twentieth Informational Supplement, M100- S20 2018; 30(1).

Easwaran S, Chittur Y R, Ramaswamy R. A study on detection of extended-spectrum betalactamases (ESBLs) and comparison of various phenotypic methods of AmpC detection in $P$. aeruginosa from various clinical isolates in a tertiary care teaching hospital. Muller J Med Sci Res. 2016; 7:359.

Gupta R, Malik A, Rizvi M, Ahmed M. Presence of metallo-beta-lactamases (MBL), extended-spectrum beta-lactamase (ESBL) \& AmpC positive non-fermenting Gramnegative bacilli among Intensive Care Unit patients with special reference to molecular detection of blaCTX-M \& blaAmpC genes. Indian J Med Res. 2016 ;144(2):271.

Haider M, Rizvi M, Fatima N, Shukla I and Malik A. Necessity of detection of extended spectrum beta- lactamase, AmpC and metallo-beta-lactamases in Gram- negative bacteria isolated from clinical specimens. Muller J Med Sci Res. 2014; 5:23-8.

Kumar H, Arora D R, Mishra B, Dogra V. Evaluation of phenotypic and genotypic detection method of metallo beta lactamase producing Pseudomonas aeruginosa. Int $\mathrm{J}$ App Basic Med Res. 2018;2:647-59.

Kumar R, Srivastava P, Rishi S. et al., Detection and antimicrobial Susceptibility pattern of
Pseudomonas aeruginosa isolates in various clinical samples with special reference to metallo beta lactamase from a tertiary care hospital in Jaipur, India. National Journal of Medical Research.2014; 4 (2): 128-31.

Padiyath S, Hemachandra C, Rao P S, Kotigadde $S$. Detection of Extended spectrum $\beta$ lactamase, AmpC $\beta$ - lactamase and Metallo $\beta$-lactamase in clinical isolates of $P$. aeruginosa. J Pharm Biomed Sci. 2013; 33: 1506-15.

Ranjan S, Banashankari G S, Sreenivasa Babu P R. Comparison of Epidemiological and Antibiotic Susceptibility Pattern of MetalloBeta-Lactamase Positive and Metallo-BetaLactamase-Negative Strains of $P$. aeruginosa. J. Lab Physicians. 2014; 6:10913.

Shaikh S, Jamale Fatima J, Shakil S, Mohd. S, Rizvi D, Mohammad A K. Prevalence of multidrug resistant and extended spectrum beta-lactamase producing $P$. aeruginosa in a tertiary care hospital. Saudi J Biol Sci. 2015; 22: 62-4.

Sharma M, Yadav S, Chaudhury U. Metallo beta lactamase producing Pseudomonas aeruginosa in neonatal septicaemia. J Lab Physicians. 2010; 2 (1): 14-16.

Senthamarai S, Reddy S K A, Sivasankari S, Anitha C, Somasunder V, Kumudhavathi M $\mathrm{S}$, Amshavathani $\mathrm{S} \mathrm{K}$, Venugopal $\mathrm{V}$. Resistance Pattern of $P$. aeruginosa in a Tertiary Care Hospital of Kanchipuram, Tamil Nadu, India. J Clin Diagn Res 2014 ; 8:30-2.

Tankhiwale S. Beta-lactamases in $P$. aeruginosa. A threat to clinical therapeutics. CurrPediatr Res. 2016; 20:253-7.

\section{How to cite this article:}

Ashna Bhasin, Poonam Loomba, Abha Sharma, Bibhabati Mishra and Ashish Bajaj. 2021. Coproduction of Extended Spectrum, AmpC and Metallo $\beta$ - Lactamases in Pseudomonas aeruginosa Isolates from a Super Speciality Center. Int.J.Curr.Microbiol.App.Sci. 10(10): 176184. doi: https://doi.org/10.20546/ijcmas.2021.1010.020 